\title{
Situational Attributes of an Occasion in which Health Literacy is Relevant
}

Janine Bröder, Orkan Okan, Torsten M. Bollweg, Dirk Bruland, Paulo Pinheiro, Ullrich Bauer

\section{Source}

Janine Bröder, Orkan Okan, Torsten M. Bollweg, Dirk Bruland, Paulo Pinheiro, Ullrich Bauer. (2019). Child and Youth Health Literacy: A Conceptual Analysis and Proposed Target-Group-Centred Definition. IJERPH, vol. 16 (18), 3417.

Namely, characteristics and demands of a given environment in which health literacy interactions-specifically the interaction with information or the health care settingtake place and that promote or hinder children in making use of individual and social health literacy assets. This, in turn, influences their agency and their real opportunities to practise and engage in health literacy interactions in their everyday lives. 